\title{
Image Colorization By Capsule Networks
}

\author{
Gökhan Özbulak ${ }^{1,2}$ \\ ${ }^{1}$ Istanbul Technical University \\ ${ }^{2}$ The Scientific And Technological Research Council Of Turkey \\ gokhan.ozbulak@\{itu.edu.tr,tubitak.gov.tr\}
}

\begin{abstract}
In this paper, a simple topology of Capsule Network (CapsNet) is investigated for the problem of image colorization. The generative and segmentation capabilities of the original CapsNet topology, which is proposed for image classification problem, is leveraged for the colorization of the images by modifying the network as follows: 1) The original CapsNet model is adapted to map the grayscale input to the output in the CIE Lab colorspace, 2) The feature detector part of the model is updated by using deeper feature layers inherited from VGG-19 pre-trained model with weights in order to transfer low-level image representation capability to this model, 3) The margin loss function is modified as Mean Squared Error (MSE) loss to minimize the image-to-image mapping. The resulting CapsNet model is named as Colorizer Capsule Network (ColorCapsNet). The performance of the ColorCapsNet is evaluated on the DIV2K dataset and promising results are obtained to investigate Capsule Networks further for image colorization problem.
\end{abstract}

\section{Introduction}

Image colorization is the problem of converting the image from grayscale to the another colorspace so that the image is colorized. As the colorization problem requires a mapping from the simpler data (one-channel grayscale image) to the more complex data (multi-channel composite image), many different mappings may be obtained with the most of them is far from satisfactory colorization. Hence, the problem of image colorization is stated as ill-posed.

There are many methods proposed in the literature to tackle down the ill-posed nature of the colorization problem, These studies may be classified as in two categories: Colorization with 1) guidance [4, 7, 10, 13, 16], and 2) no guidance [2, 3, 8, 12, 17]. In guided colorization, user interaction/example image is asked to provide feedback about colorization and this feedback is combined with the colorization algorithm to obtain satisfactory results. In col-

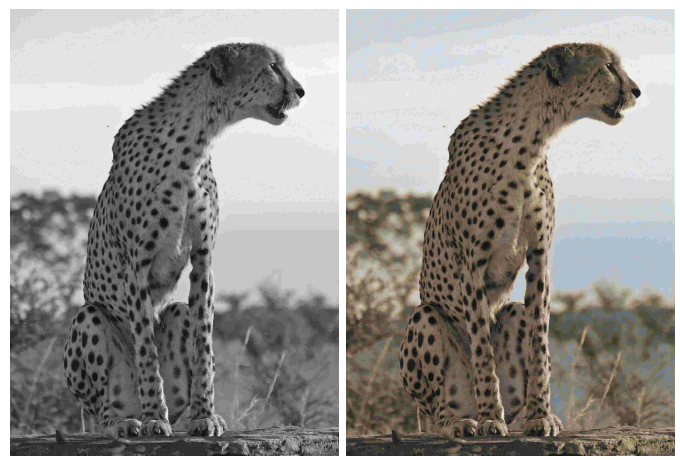

Figure 1: An example of colorization by the ColorCapsNet (psnr=23.74,ssim=0.91).

orization with no guidance, automatic colorization algorithms are considered with excluding user intervention. The latter approach is harder to get satisfactory colorization results because fully automatic methods may fail to decide for the proper colors when the alternative colors are possible for the object in interest.

In recent years, with the advancements in deep learning, many convolutional and generative deep models have arised to tackle down the challenging image analysis problems varying from object classification and detection to enhancement (denoising, colorization etc.). Especially, the problem of automatic image colorization is massively investigated by leveraging successful deep models and promising results are obtained quantitatively and qualitatively with these models.

A recent study, which proposes a deep architecture named as Capsule Network (CapsNet) [14], introduces a network capable both of image classification and generation. The experimental results of proposed method in [14] show that state-of-the-art (SOTA) performance may be obtained on image classification task by using a shallow CapsNet architecture. On the other hand, the image generation/latent space representation capability remains an open research area for further investigation.

In this paper, the generative characteristic of the CapsNet 
model is considered for the image colorization problem. The original architecture proposed in [14] consists of one feature detection layer (convolutional layer), one feature representation layer (primary capsule layer) and one classification layer (capsule layer), and is trained for the digit classification task. Here, first, the capacity of the feature detector is increased by adding more convolutional layers. The resulting feature detector is same as the first two convolutional layers of VGG-19 model [15]. For these layers, the weights from VGG-19 model pre-trained on ILSVRC 2012 [5] are also transferred before training in order to initialize the network with prior low-level feature representation. This may be regarded as a transfer learning strategy. Then, the network is adapted to generate the image in CIE Lab colospace from its grayscale counterpart. Finally, the margin loss, which is defined for the classification task, is changed to Mean Squared Error (MSE) loss in order to minimize the difference between real and generated color images. The resulting colorization model is named as Colorizer Capsule Network (ColorCapsNet). A colorization example by ColorCapsNet can be examined in Figure 1.

ColorCapsNet is trained on two different datasets. First, it's trained on ILSVRC 2012 dataset [5] in order to learn the general color distribution of the objects. Then, DIV2K dataset [1] is used to obtain final colorization model. In both cases, the datasets are pre-processed for proper colorspace conversion and data size. Proposed method is patch-based so that a pre-defined patch size must be determined before training. The patch-based mapping exploits the information in local neighborhood so that the pixel in interest is colorized according to the color distribution in its neighborhood [2].

The organization of the paper is as follows: In Section 2 , the literature is reviewed for both image colorization with guidance and no guidance. In Section 3 , the ColorCapsNet model is explained in detail. The performance of the method is discussed in Section 4 and the paper is concluded in Section 5 with possible future directions.

\section{Related Work}

In the literature, the image colorization problem is mainly considered in two categories as follows.

Colorization With Guidance. In this approach, a user interaction or a set of guidance pixels are asked to provide a prior information to the colorization system in order to obtain realistic results. In [13], user provided scribbles are used to colorize the neighboring pixels that have similar intensity values. This method eliminates the need of the object segmentation and considers the problem as an optimization procedure that minimizes a quadratic cost function. The method proposed in [10] uses example image segments and transfers the colors from segments into the grayscale areas by keeping the spatial coherency high.
An interactive colorization algorithm, which is proposed in [16], assigns weights for user scribbles and combines them for final colorization. In [4], the user is asked to provide the localization and labelling for the salient foreground object to be colorized and then the object is colorized by using reference images retrieved from internet. And [7] transfers the color information into the grayscale images from semantically similar reference images by using superpixel representation to speed up the colorization and to have better spatial consistency. The main drawback of the guided colorization algorithms is that they are highly dependent to user feedback or reference example images and this causes the algorithms to fail generalizing well for all type of colorizations.

Colorization With No Guidance. The methods that apply colorization without any feedback fall into this category and they colorize the given grayscale images automatically in an end-to-end fashion. In [2], the grayscale pixels are considered at local and global levels by estimating the multimodal color distribution and applying graph-cut algorithm respectively. The method in [3] proposes a deep colorization model combined with adaptive image clustering and joint bilateral filter for global consideration and colorization enhancement respectively. In [12], as similar to [2, 3], a deep model is trained to generate the per-pixel color histogram that represents the multimodal color distribution. The method in [8] proposes an end-to-end CNN model that combines the local features with global information. In [17], as similar to [8], a CNN model is constructed with the class-rebalancing mechanism in order to provide the color diversity for the ill-posed nature of the colorization problem. The automatic colorization methods provide end-to-end fully colorization mechanisms while they may be lack of solving the ambiguity in case of multiple choice of colorization.

\section{Proposed Method}

In this section, the design of the ColorCapsNet model is explained in detail. First, the pre-processing steps such as colorspace selection and data preparation are explained. Then, the modifications on topology and optimization procedures are presented. Finally, the selection of critical parameters, which affects the colorization performance, are discussed.

\subsection{Data Pre-processing}

Colorspace. In this study, as in [2, 7, 12, 17], CIE Lab colorspace is used to represent the color images. CIE Lab is made of three channels as $\mathrm{L}$, a an b. L represents the lightness/luminance whereas $\mathrm{a}$ and $\mathrm{b}$ are the chrominance. CIE Lab is a perceptually linear colorspace as it establishes a mapping between the colors in Euclidean space and the colors in human perception. Therefore, it's more suitable 
than other colorspaces for colorization task and preferred to represent the colors in this study as well.

Data Representation. The ColorCapsNet maps a grayscale image patch to the corresponding color image patch in CIE Lab colorspace. Therefore, during both training and testing phases, the data must be fed into the ColorCapsNet accordingly. For training phase, first, each color image given in RGB colorspace is converted into CIE Lab colorspace. Then, both the color and corresponding grayscale images are sliced into the $n x n$ square patches, where $n$ is a pre-defined value (see Section 3.4), and stacked as image patch pairs. In testing phase, the test image in grayscale is sliced as same in training and fed into the trained model for colorization. The result is a stack of the predicted color patches in CIE Lab colorspace and the patches are put together to make the resulting image complete. Finally, the estimated image is converted from CIE Lab into RGB for visual perception. The flow for data preprocessing is illustrated in Figure 2 .

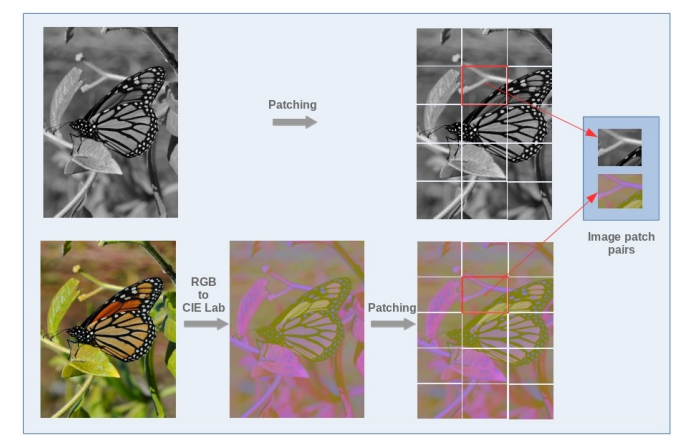

Figure 2: Data pre-processing flow.

\subsection{Network Design}

The first modification is on the feature detection part of the network. In the original CapsNet [14], this part consists of one convolutional layer with 256 filters. These filters have size of 9x9 with stride of 1 and are activated with Rectified Linear Unit (ReLU) to feed the feature representation layer named as primary capsule. In proposed method, the feature detector is replaced with the first and the second convolutional layers of the VGG-19 model [15] and both layers are identical as they have 64 convolution filters with size of $3 \times 3$, stride of 1 and ReLU activation. These convolutional layers, on contrary to the rest of the network, are initialized by transferring the weights of first two layers from the pre-trained VGG-19 model without any freezing process. Such an initialization can be regarded as a transfer learning strategy, and, here, the purpose is to transfer the low level feature representation capability of the VGG-19 model into the ColorCapsNet so that it may detect the low level features (corners, edges etc.) as good as VGG-19. The performance of detecting low level features has impact on the object segmentation that further affects the colorization quality. As seen in Figure 4a, such modification reduces the train loss from 0.0035 to 0.0033 after 10 epochs training. In this case, the total number of trainable parameters also reduces by 720000 approximately.

The second modification is to add Batch Normalization (BN) layer after each convolutional layer. The reason of adding BN layer is to reduce the effect of the Internal Covariate Shift (ICS) problem stated in the study [9] that proposes $\mathrm{BN}$ as a solution to this problem. There are two benefits of applying BN: 1) It speeds up the training process, 2) It regularizes the network for better generalization.

BN normalizes the training instance, $x_{i}$, in the minibatch $B=\left\{x_{1}, . ., x_{b}\right\}$, which is a small representation of train set with the size of $b$, so that it has zero mean with unit variance $\left(\mu=0, \sigma^{2}=1\right)$. In order to make the ColorCapsNet ICS-reduced, first, the mean is calculated for the mini-batch $B$ of the patch pairs as below:

$$
\mu_{B}=\frac{1}{b} \sum_{i=1}^{b} x_{i}
$$

Then, the variance of $B, \sigma_{B}^{2}$, is calculated:

$$
\sigma_{B}^{2}=\frac{1}{b} \sum_{i=1}^{b}\left(x_{i}-\mu_{B}\right)^{2}
$$

Finaly, $B$ is normalized to zero mean with unit variance to reduce the ICS:

$$
\hat{x}_{i}=\frac{x_{i}-\mu_{B}}{\sqrt{\sigma_{B}^{2}}}
$$

$\mathrm{BN}$ is applied to the first two convolutional layers of the feature detection part and the right after the convolutional layer in the primary capsule part. The effect of applying $\mathrm{BN}$ can be examined in Figure $4 \mathrm{a}$

The third modification is for the number of capsule in the classification layer (capsule layer). In the original CapsNet topology, the number of capsule is selected as 10 to address the 10-class digit classification task. Here, because the colorization is an image generation task rather than classification, the parameter of the capsule number in the capsule layer should be adapted to this problem. For this parameter, moving from 10 to 6 doesn't change the loss so much as seen in Figure 4 a but it dramatically reduces the number of trainable parameters by 2390000 approximately. Therefore, the number of the capsule is selected as 6 in the capsule layer.

The resulting topology is illustrated in Figure 3 As seen in the figure, the topology resembles an autoencoder that is made of an encoder and decoder network. There is a vector representation, which is defined in latent space, between 


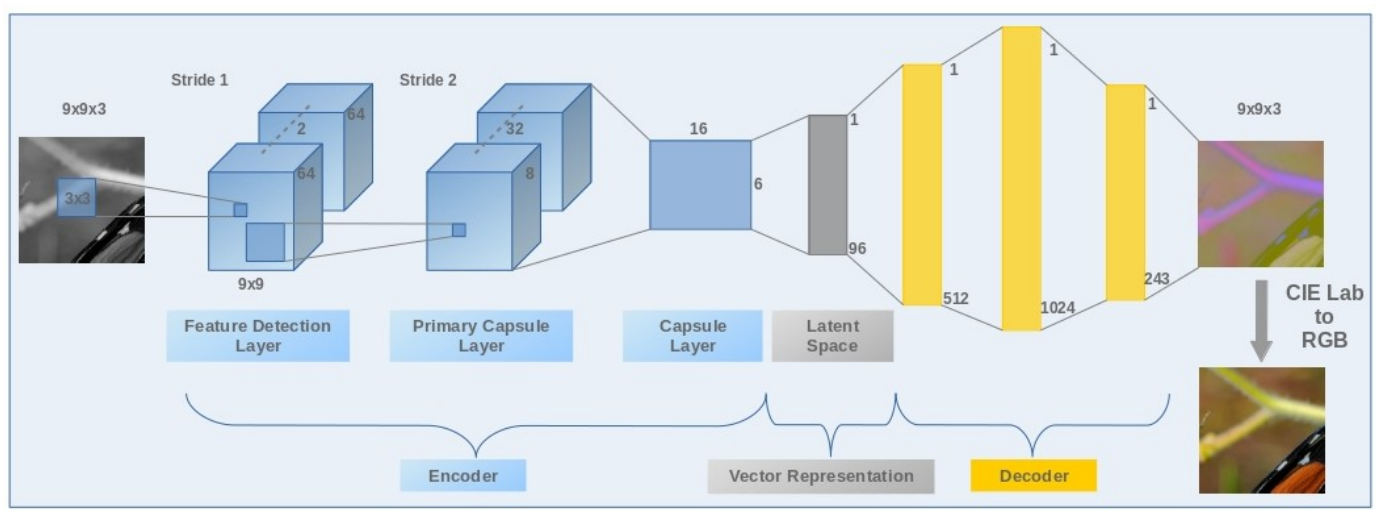

Figure 3: The ColorCapsNet topology.

encoder and decoder networks and it represents the hidden variables related with colorization task.

\subsection{Optimization Procedure}

In the original CapsNet model, the margin loss is used to minimize the loss during training. The margin loss is defined for each capsule in the classification layer (capsule layer) as below:

$L_{c}=T_{c} \max \left(0,0.9-\left\|v_{c}\right\|\right)^{2}+\lambda\left(1-T_{c}\right) \max \left(0,\left\|v_{c}\right\|-0.1\right)^{2}$

where $\lambda=0.5, v_{c}$ is the output of the capsule $c$ and $T_{c}=1$ when the capsule representing the class is activated. The total loss over all class capsules are as follows:

$$
L=\sum_{c=1}^{C} L_{c}
$$

In the ColorCapsNet mode, because the optimization is to minimize the difference between real and generated color images, the objective function for loss is defined as Mean Squared Error (MSE) as below:

$$
M S E=\frac{1}{Y X} \sum_{y=1}^{Y} \sum_{x=1}^{X}[I(x, y)-\hat{I}(x, y)]^{2}
$$

where $I(x, y)$ and $\hat{I}(x, y)$ are the corresponding real and generated color image pixel values respectively. As stated in [17], $L_{2} / M S E$ is the proper loss function for CIE Lab colorspace because it defines the chroma in the Euclidean space and it's effective to minimize $M S E$ in this space.

As in the CapsNet, the Adam optimizer [11] is leveraged as the optimization method during forward-backward pass with the learning rate of $0.001, \beta_{1}$ of 0.9 and $\beta_{2}$ of 0.999 .

\subsection{Parameter Selection}

Number of Routings. The routing is one of the most critical hyperparameters in the CapsNet topology. The routing is an iterative procedure for information transfer between the capsules in different layers. In the routing mechanism, the capsule in the lower layer is connected to the activated capsule in the upper layer for transferring its output. This mechanism is called as "routing-by-agreement". According to [14], the "routing-by-agreement" leverages the information about the shape of the object in pixel level so that the segmentation could be achieved while moving from locality to global extent. The ColorCapsNet also exploits the idea of routing in order to segment objects internally and to colorize them. The number of the iterations during routing agreement should be selected carefully in order to achieve proper information transfer between capsules in different layers. In [14], the best performance is obtained by routing 3 times. In Figure $4 \mathrm{~b}$, it can be observed that the change in the number of routing doesn't effect the colorization performance. The drawback of using iterative routing is that it increases the time complexity of training as the number of routing goes up. By considering this fact, the number of routings is selected as 1 in order to accelerate the training process.

Patch Size. The size of patch is another critical parameter for successful colorization because it defines the boundary of the local structures and leads to better understanding of exposing them. In this study, three different patch sizes are considered for colorization task: 9x9, 16x16 and 32x32. $9 \times 9$ and $32 \times 32$ are selected as the minimum and the maximum patch sizes respectively because $9 \times 9$ is the theoretical lower bound as input and the patch sizes bigger than $32 \times 32$ cause visual discontinuities at border of adjacent patches. In Figure 4c it's obviously seen that the train loss decreases with the patch size goes down exponentially. Therefore, the patch size is selected as $9 \times 9$ for this problem. 


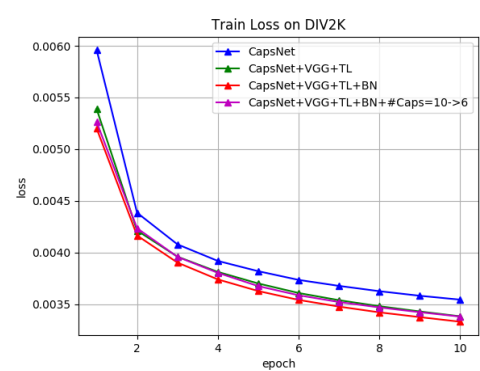

(a) Topology

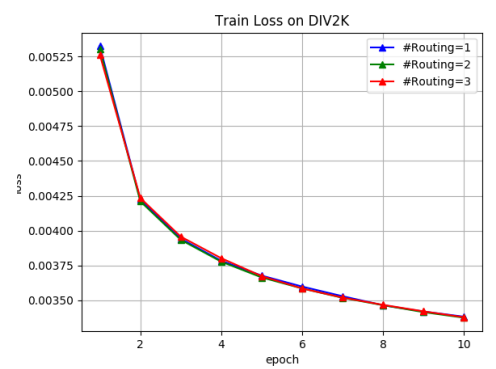

(b) Number of routings

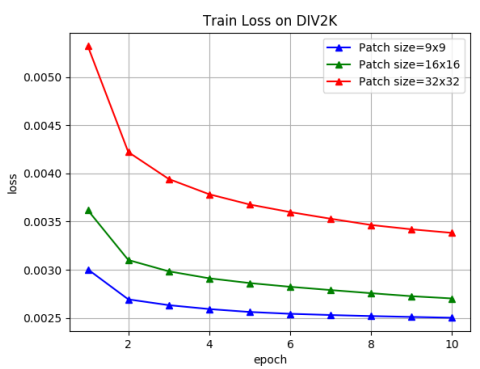

(c) Patch size

Figure 4: Comparative analysis for network design of the ColorCapsNet.

\section{Experimental Analysis}

In this section, the performance of the ColorCapsNet is quantitatively evaluated by testing with validation and test sets from DIV2K dataset. The results are reported with well-known evaluation metrics, PSNR and SSIM. Some perceptual results are also shown from validation and test sets to demonstrate the effectiveness of the ColorCapsNet on the fully automatic colorization task.

\subsection{Datasets}

ILSVRC 2012 [5] and DIV2K [1] are two datasets used in this study to model the colorization. The reason of using ILSVRC 2012 is to supervise the ColorCapsNet with general color distribution of the objects in the dataset. This helps the network to have prior information about object colors before trained on DIV2K. Once, the ColorCapsNet is trained on ILSVRC 2012, as next step, DIV2K dataset is used for training to get the final colorization model. Both datasets are pre-processed to have colorized-grayscaled image pairs in pre-defined patch size.

The validation and test sets of the ILSVRC 2012 dataset are used in this study as follows: First, both sets are merged with ending up 150000 RGB images. Then, these 150000 images are converted into the images in grayscale and CIE Lab. Finally, the corresponding grayscale and CIE Lab images are divided into the $9 \times 9 \times 3$ patches to train the network. The total number of patches is 26536446.

DIV2K dataset has 794 grayscale and 800 RGB images in the train set, and it has 100 grayscale/RGB pairs in the validation set. First, 794 out of 800 images in the train set are used for consistency and there is only RGB to CIE Lab conversion because corresponding grayscale images are provided. Grayscale/CIE Lab image pairs are again divided into 9x9x3 patches with the total number of 6788644 image pairs. The pre-trained network on ILSVRC 2012 is trained on this dataset for estimating the images from validation set (The estimations of the validation images in Figure 6 are based on this model). Then, 100 grayscale/RGB pairs from validation set are considered in same manner with ending up 869196 image pairs as 9x9x3 patches. The pre-trained network on train set of DIV2K is finally trained on this validation set to colorize the test images (The test images in Figure 6 are colorized with this model).

\subsection{Evaluation Metrics}

In image colorization domain, the Peak Signal to Noise Ratio (PSNR) and the Structural Similarity Index Measure (SSIM) are two widely used evaluation metrics to show the effectiveness of the colorization operation. In this study, the colorization performance is also evaluated with these metrics.

PSNR is the ratio between the power of the peak signal and the power of the noisy signal in terms of the logarithmic scale. It's formulated as below:

$$
P S N R=10 \log _{10}\left(\frac{\text { peak }}{M S E}\right)
$$

where peak is the power of the peak signal and $M S E$ is the mean squared error between the original and the noise signals. The bigger PSNR values mean to the better quality and the lower noise in the image. Similarly, in the domain of the image colorization, the bigger PSNR indicates the better colorization performance.

SSIM is another image quality metric and used to measure the similarity between real and estimated images based on luminance, contrast and structure. It's formulated as below:

$$
\operatorname{SSIM}(I, \hat{I})=\frac{\left(2 \mu_{I} \mu_{\hat{I}}+C_{1}\right)\left(2 \sigma_{I \hat{I}}+C_{2}\right)}{\left(\mu_{I}^{2}+\mu_{\hat{I}}^{2}+C_{1}\right)\left(\sigma_{I}^{2}+\sigma_{\hat{I}}^{2}+C_{2}\right)}
$$

where $\mu$ is the mean, $\sigma$ is the standard deviation, $\sigma_{I \hat{I}}$ is the covariance and $C_{1}$ and $C_{2}$ are the contrast related parameters. The bigger SSIM indicates the better reconstruction of the real image by the estimated one. For the image colorization, the bigger SSIM value means to the better colorization result. 


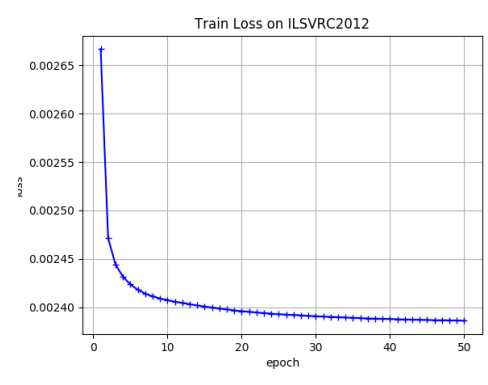

(a) Train loss on ILSVRC 2012

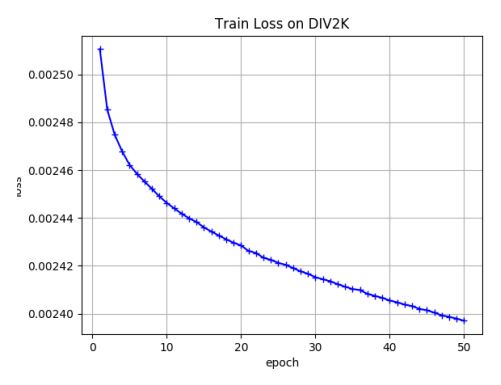

(b) Train loss on DIV2K train set

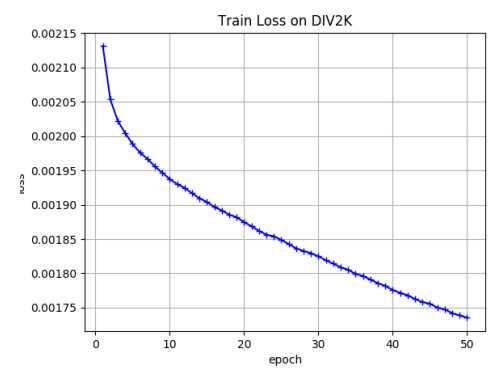

(c) Train loss on DIV2K validation set

Figure 5: The train loss performance of the ColorCapsNet.

\subsection{Results}

The loss performance during training on ILSVRC 2012, DIV2K train and validation sets is shown in Figure 5, Although the training on ILSVRC 2012 is saturated in 50 epochs (see Figure 5a), there is still chance to decrease the loss after 50 epochs for the training on DIV2K dataset (Figure $5 \mathrm{~b}$. Figure $5 \mathrm{c}$.

For the validation and test phases of the NTIRE 2019 Colorization Challenge [6], the comparative performance of the ColorCapsNet with other methods can be examined in Table 1. Proposed method, the ColorCapsNet, has comparable colorization performance on both phases although it's just a shallow CapsNet architecture and doesn't contain any complex mechanism for some important problems such as spatial coherency. The validation performance for the ColorCapsNet trained in 50 epochs is as PSNR of 22.20 and SSIM of 0.88. The test performance in 10 epochs is as PSNR of 21.08 and SSIM of 0.85 (The testing in 50 epochs couldn't be evaluated because of the unavailability of the colorized test data). The worst and the best performances are listed in Table 1 as well independently for PSNR and SSIM values. In other word, these values are independently the worst and the best values that may or may not belong to the same participant.

As seen from Figure $5 \mathrm{~b}$ and Figure $5 \mathrm{c}$. it's also possible to continue training on DIV2K for better fitting because there is no elbow in the plots after 50 epochs so that it has still capacity to converge further. The original CapsNet model [14] is trained in 1250 epochs with the sign of the convergence at 500 epochs for the digit classification task.

Some colorization results can be examined in Figure 6 According to the visual results, it can be said that the ColorCapsNet has promising colorization capability without any guidance. Even for some colorization results with low PSNR and SSIM values (Figure 6f and Figure 6j), it may generate satisfactory results. Those results in Figure 6 with PSNR and SSIM values of ' $n / a$ ' indicate that there was no real color image corresponding to the estimated one and the

\begin{tabular}{|l|c|c|c|}
\hline Method & Metric & Validation & Test \\
\hline \hline Worst & & $16.39 / 0.55$ & $17.96 / 0.84$ \\
Best & PSNR/ & $22.73 / 0.93$ & $22.19 / 0.94$ \\
ColorCapsNet & SSIM & $22.20 / 0.88^{1}$ & $21.08 / 0.85^{2}$ \\
\hline
\end{tabular}

Table 1: Validation and test results in the NTIRE 2019 Colorization Challenge [6] $\left({ }^{1}: 50\right.$ epochs $,{ }^{2}: 10$ epochs $)$.

evaluation metrics couldn't be calculated.

In Figure 7, the progress in the colorization during training can be examined for two instances from the DIV2K validation set. As seen in the figure, although the PSNR values oscillate softly with the changing number of the epoch (24.38 \pm 0.56 for the top row and $23.65 \pm 0.09$ for the bottom row), the SSIM values are stabilized as indicating no more improvement on the colorization performance. The effect of the longer training on the colorization performance should be further investigated in order to see if it helps to the improvement as in [14].

\section{Conclusion}

In this paper, a simple Capsule Network (CapsNet) architecture is designed for ill-posed image colorization problem. Proposed method is a fully automatic, end-to-end, patch-based deep model named as Colorizer Capsule Network (ColorCapsNet) and it exploits the generative and segmentation capabilities of the CapsNets for the colorization task. Experiments show that the ColorCapsNet has promising and comparable results on provided DIV2K dataset with its simple design and deserves further investigation such as designing deeper CapsNet architectures or integrating multimodal color distribution for better colorization performance. 


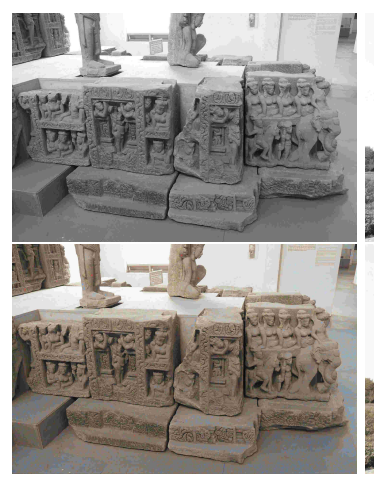

(a) $\mathrm{psnr}=28.46, \mathrm{ssim}=0.92$

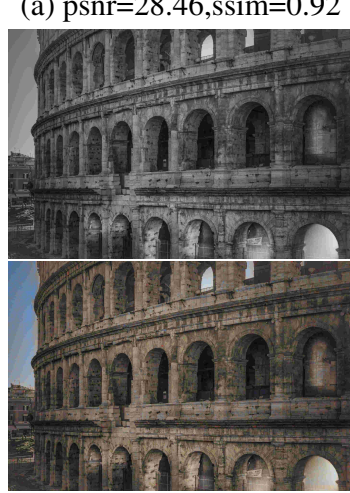

(e) $p s n r=26.40$, ssim $=0.95$

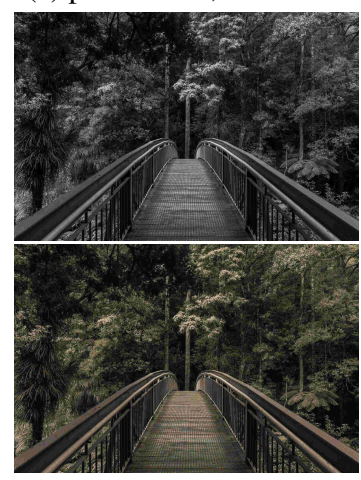

(i) $\mathrm{psnr}=26.92, \mathrm{ssim}=0.91$

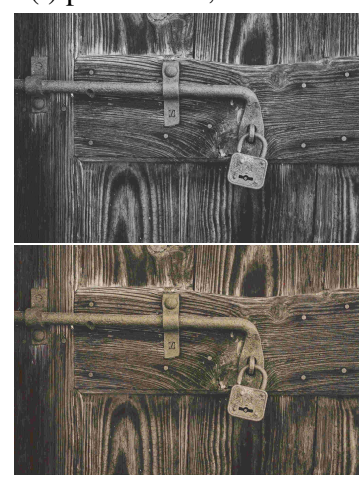

(m) $\mathrm{psnr}=23.89, \mathrm{ssim}=0.93$

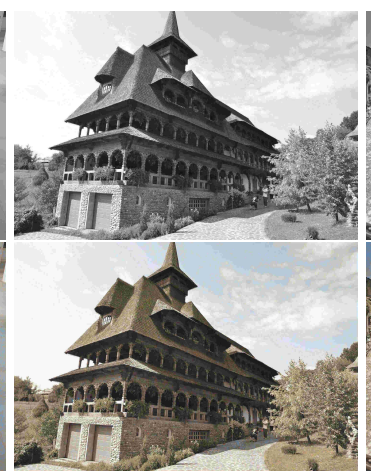

(b) $\mathrm{psnr}=23.26, \mathrm{ssim}=0.94$

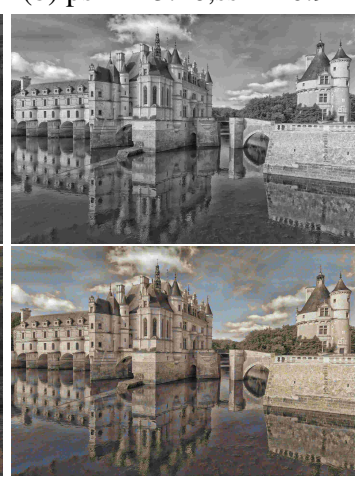

(f) $\mathrm{psnr}=21.98, \mathrm{ssim}=0.89$

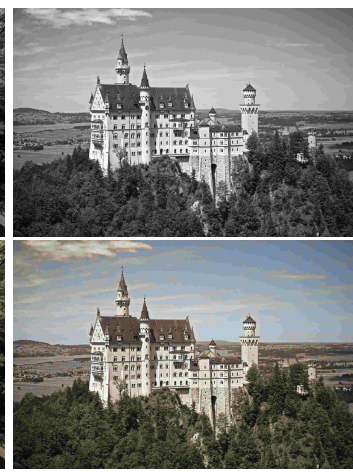

(j) $p s n r=19.52, \mathrm{ssim}=0.86$

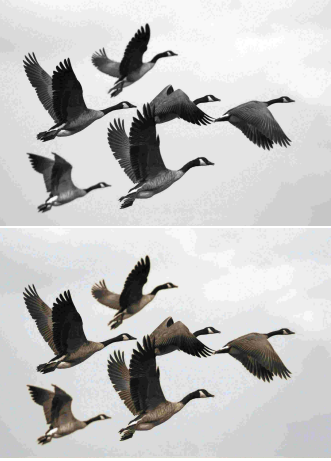

(n) $\mathrm{psnr}=30.07, \mathrm{ssim}=0.94$

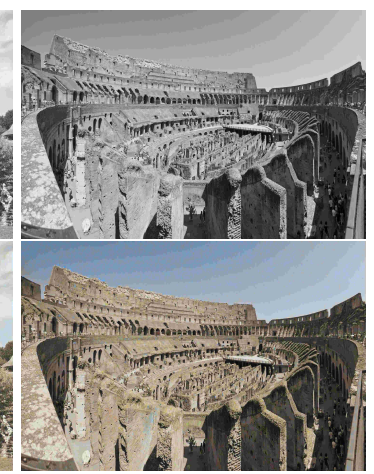

(c) $\mathrm{psnr}=26.58, \mathrm{ssim}=0.94$

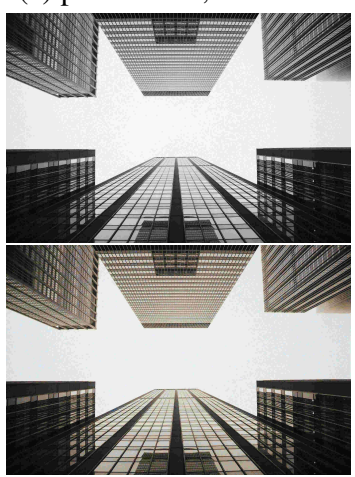

(g) $\mathrm{psnr}=25.73, \mathrm{ssim}=0.93$

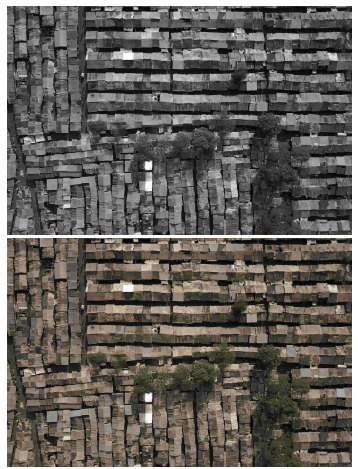

(k) $\mathrm{psnr}=23.61, \mathrm{ssim}=0.91$

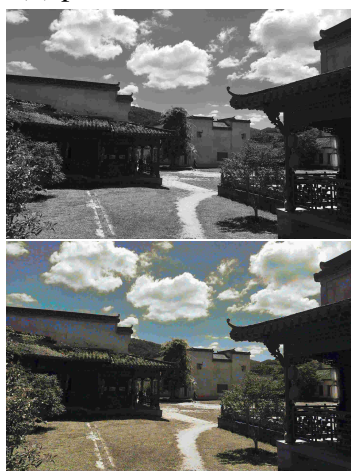

(o) $\mathrm{psnr}=\mathrm{n} / \mathrm{a}, \mathrm{ssim}=\mathrm{n} / \mathrm{a}$

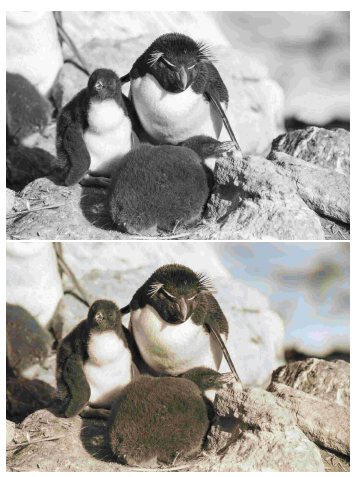

(d) $\mathrm{psnr}=26.57, \mathrm{ssim}=0.95$

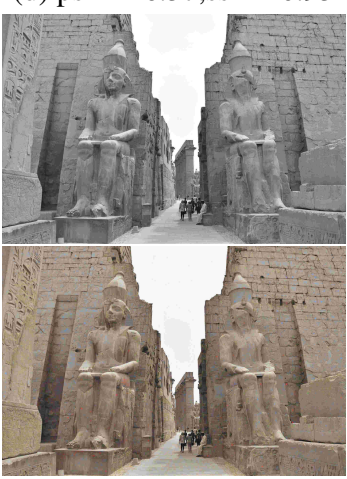

(h) $\mathrm{psnr}=28.26, \mathrm{ssim}=0.94$

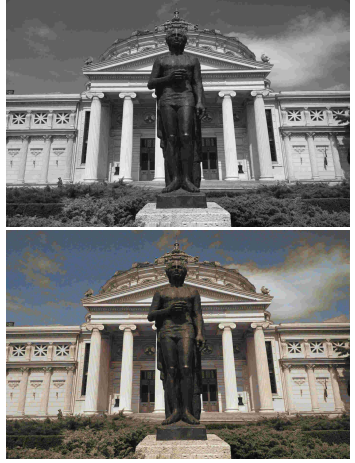

(1) $\mathrm{psnr}=23.56, \mathrm{ssim}=0.91$

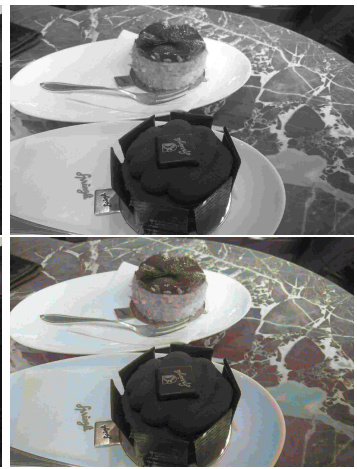

(p) $\mathrm{psnr}=\mathrm{n} / \mathrm{a}, \mathrm{ssim}=\mathrm{n} / \mathrm{a}$

Figure 6: Some colorization results from DIV2K dataset. 


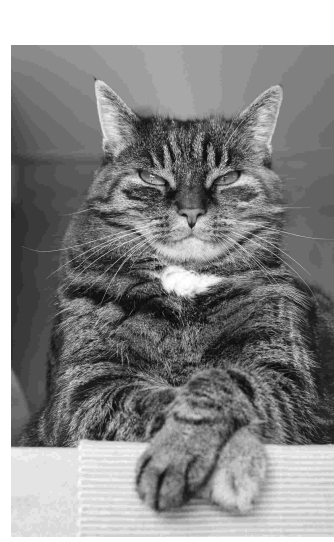

(a) Grayscale

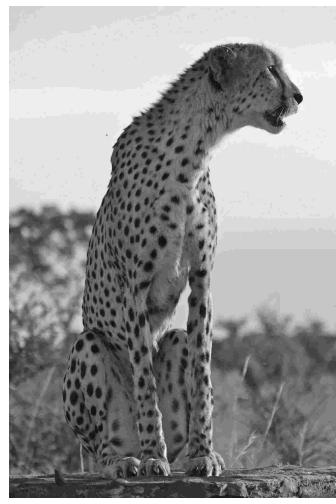

(f) Grayscale

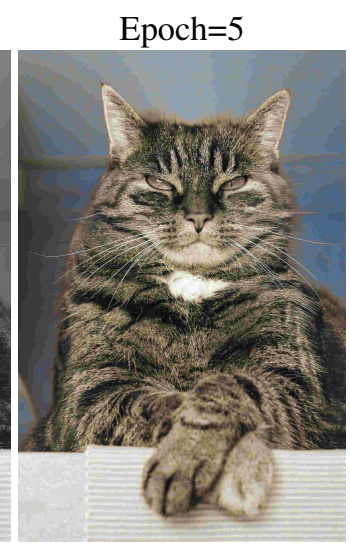

(b) $\mathrm{psnr}=24.73, \mathrm{ssim}=0.92$

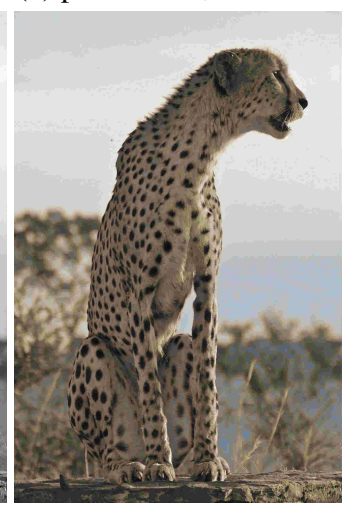

(g) psnr=23.60, ssim $=0.92$

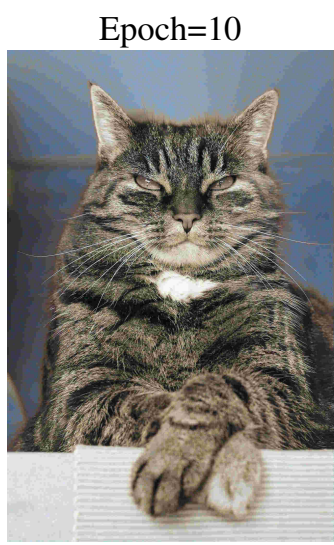

(c) $\mathrm{psnr}=23.71, \mathrm{ssim}=0.92$

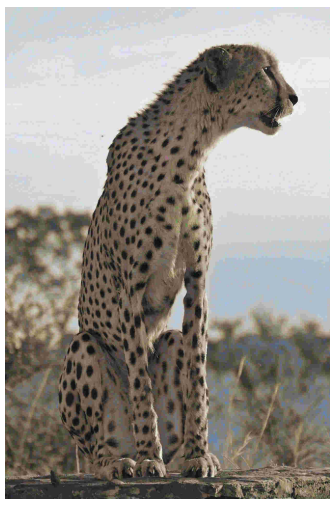

(h) $\mathrm{psnr}=23.53, \mathrm{ssim}=0.91$

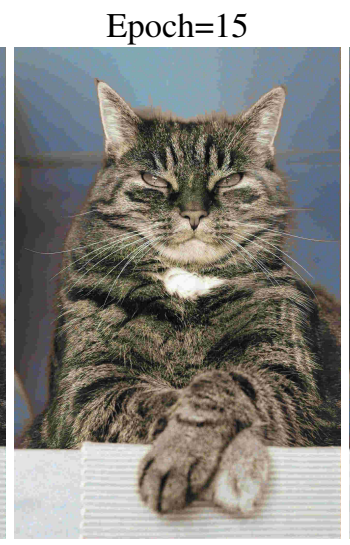

(d) $\mathrm{psnr}=23.97, \mathrm{ssim}=0.92$

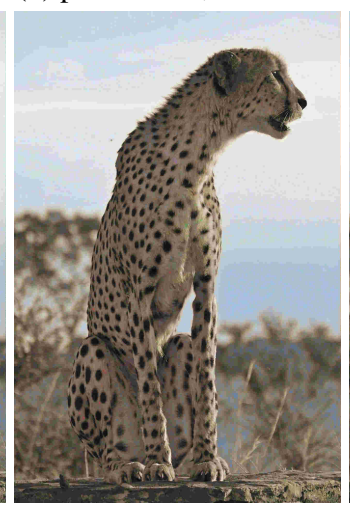

(i) $\mathrm{psnr}=23.75, \mathrm{ssim}=0.91$

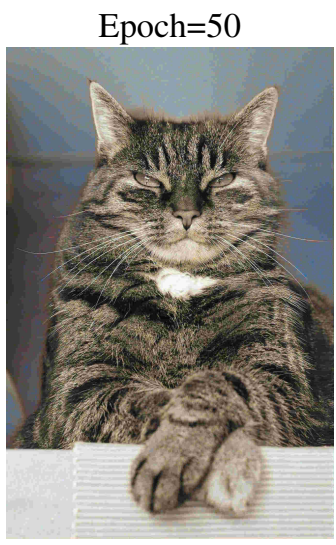

(e) $\mathrm{psnr}=25.11, \mathrm{ssim}=0.92$

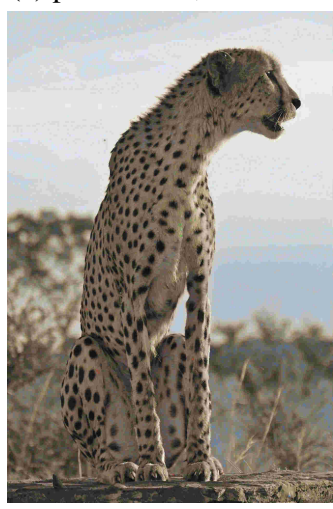

(j) $p s n r=23.74$, ssim $=0.91$

Figure 7: Colorization progress across training.

\section{References}

[1] Eirikur Agustsson and Radu Timofte. Ntire 2017 challenge on single image super-resolution: Dataset and study. In Proceedings of the IEEE Conference on Computer Vision and Pattern Recognition Workshops, pages 126-135, 2017.

[2] Guillaume Charpiat, Matthias Hofmann, and Bernhard Schölkopf. Automatic image colorization via multimodal predictions. In European conference on computer vision, pages 126-139. Springer, 2008.

[3] Zezhou Cheng, Qingxiong Yang, and Bin Sheng. Deep colorization. In Proceedings of the IEEE International Conference on Computer Vision, pages 415-423, 2015.

[4] Alex Yong-Sang Chia, Shaojie Zhuo, Raj Kumar Gupta, YuWing Tai, Siu-Yeung Cho, Ping Tan, and Stephen Lin. Semantic colorization with internet images. In ACM Transactions on Graphics (TOG), volume 30, page 156. ACM, 2011.

[5] Jia Deng, Wei Dong, Richard Socher, Li-Jia Li, Kai Li, and Li Fei-Fei. Imagenet: A large-scale hierarchical image database. In 2009 IEEE conference on computer vision and pattern recognition, pages 248-255. Ieee, 2009.

[6] Shuhang Gu, Radu Timofte, Richard Zhang, et al. Ntire 2019 challenge on image colorization: Report. In The IEEE Conference on Computer Vision and Pattern Recognition (CVPR) Workshops, June 2019.
[7] Raj Kumar Gupta, Alex Yong-Sang Chia, Deepu Rajan, Ee Sin $\mathrm{Ng}$, and Huang Zhiyong. Image colorization using similar images. In Proceedings of the 20th ACM international conference on Multimedia, pages 369-378. ACM, 2012.

[8] Satoshi Iizuka, Edgar Simo-Serra, and Hiroshi Ishikawa. Let there be color!: joint end-to-end learning of global and local image priors for automatic image colorization with simultaneous classification. ACM Transactions on Graphics (TOG), 35(4):110, 2016.

[9] Sergey Ioffe and Christian Szegedy. Batch normalization: Accelerating deep network training by reducing internal covariate shift. arXiv preprint arXiv:1502.03167, 2015.

[10] Revital Ironi, Daniel Cohen-Or, and Dani Lischinski. Colorization by example. In Rendering Techniques, pages 201210. Citeseer, 2005.

[11] Diederik P Kingma and Jimmy Ba. Adam: A method for stochastic optimization. arXiv preprint arXiv:1412.6980, 2014.

[12] Gustav Larsson, Michael Maire, and Gregory Shakhnarovich. Learning representations for automatic colorization. In European Conference on Computer Vision, pages 577-593. Springer, 2016. 
[13] Anat Levin, Dani Lischinski, and Yair Weiss. Colorization using optimization. In ACM transactions on graphics (tog), volume 23, pages 689-694. ACM, 2004.

[14] Sara Sabour, Nicholas Frosst, and Geoffrey E Hinton. Dynamic routing between capsules. In Advances in neural information processing systems, pages 3856-3866, 2017.

[15] Karen Simonyan and Andrew Zisserman. Very deep convolutional networks for large-scale image recognition. arXiv preprint arXiv:1409.1556, 2014.

[16] Liron Yatziv and Guillermo Sapiro. Fast image and video colorization using chrominance blending. IEEE transactions on image processing, 15(5):1120-1129, 2006.

[17] Richard Zhang, Phillip Isola, and Alexei A Efros. Colorful image colorization. In European conference on computer vision, pages 649-666. Springer, 2016. 